César Bugallo Carrera'

Manuel Gandoy Crego ${ }^{2}$

Cristina Gómez Cantorna ${ }^{3}$

1. Psicólogo. Doctor en Gerontología. Investigador del grupo de investigación Dependencia, Gerontología y Geriatría de la USC. Departamento de Enfermería. Escuela Universitaria de Enfermería. Santiago de Compostela.

2. Profesor titular de la USC y coordinador del grupo de investigación Dependencia, Geriatría y Gerontología de la USC. Departamento de Enfermería. Escuela Universitaria de Enfermería. Santiago de Compostela.

3. Enfermera. Doctora en Gerontología. Investigadora del grupo de investigación Dependencia, Gerontología y Geriatría de la USC. Departamento de Enfermería. Escuela Universitaria de Enfermería. Santiago de Compostela.

\section{La calidad de vida de los sujetos usuarios de un centro social de personas mayores}

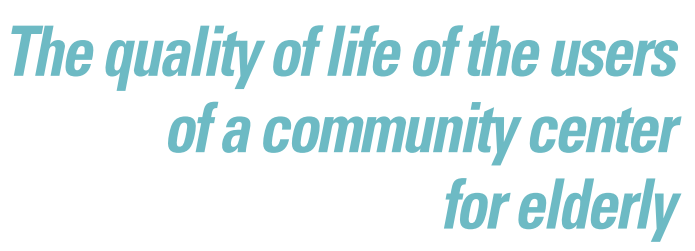

Correspondencia:

César Bugallo Carrera

c/ Ramón Caamaño 14

15124 Muxía (A Coruña)

Tel.: 645401216

E-mail: cesarbugallocarrera@gmail.com

\section{RESUMEN}

En esta investigación hemos intentado comprobar si la asistencia a los centros sociales de personas mayores es un medio eficaz para la mejora de la calidad de vida de las personas mayores, es decir, si los usuarios de dichos centros tienen una mejor calidad de vida que aquellos que no acuden a ellos. Para ello, hemos contado con un grupo experimental y un grupo control.

A continuación ambos grupos han pasado dos pruebas: una que valora la calidad de vida y otra, el deterioro cognitivo. Una vez pasadas las pruebas y obtenidas las puntuaciones, hemos concluido que los usuarios del centro social tienen una mayor calidad de vida que los sujetos que no acuden a él.

PALABRAS CLAVE: gerontología, calidad de vida, personas mayores, centros sociales.

\section{ABSTRACT}

In this investigation we have tried to verify if the assistance to the community centers of major persons is an effective way for the improvement of the major persons's quality of life, that is to say, if the users of the above mentioned centers have a better quality of life that those that do not come to them.

For it, we have possessed an experimental group and a group control. Later we have gone on to them to both groups two tests: one that values the quality of life and other one the cognitive deterioration. Once spent the tests and obtained the punctuations we have concluded that the users of the community center have a major quality of life that the subjects that do not come to it.

KEYWORDS: Gerontology, quality of life, major persons, community centers.

\section{- INTRODUCCIÓN}

Con el aumento de la esperanza de vida, gracias a los avances de las sociedades modernas, se ha producido la aparición de un colectivo cada vez más grande de personas mayores con un peso poblacional, social, económico y político cada vez mayor.

Este colectivo de edad, cada vez más amplio y mejor organizado, irrumpe con fuerza demandando respuestas para sus necesidades tanto económicas como sanitarias, educativas, sociales o de ocio.

Como respuesta a esas demandas, los poderes políticos y sociales han realizado una serie de reformas legislativas así como la creación de una serie de equipamientos para atender las necesidades de este colectivo.

En medio de este contexto es donde nacen los centros sociales de personas mayores, concebidos como recursos gerontológicos abiertos a la comunidad que se dirigen a promover el envejecimiento activo, favoreciendo el desarrollo personal, la convivencia y la participación social. Estos centros fueron concebidos en su origen como lugares de encuentro de personas mayores, cuya filosofía se centraba en proporcionar un local en el que las personas mayores se pudieran reunir para fomentar las relaciones y disfrutar del tiempo libre.

Sin embargo, en la actualidad, la oferta de actividades se ha diversificado ampliamente hasta convertir estos centros en importantes lugares de ocio y aprendizaje. El objetivo es que las personas mayores sean las protagonistas, es decir, se busca su participación en charlas, cursos y talleres, animándolos, en definitiva, a envejecer en compañía y a ampliar sus redes de relación.

Por todo ello, el objetivo principal de esta investigación es comprobar si la asistencia a los centros sociales de personas mayores es un medio eficaz para la mejora de la calidad de vida de estas personas, es decir, si los usuarios de dichos centros tienen una mejor calidad de vida que aquellos que no acuden a ellos.

Entre los objetivos específicos podemos destacar los siguientes:

- Evaluar la calidad de vida en mayores de 60 años de forma multidimensional. 
- Valorar la salud en general.

- Valorar el apoyo social.

- Evaluar las habilidades funcionales.

- Conocer la actividad y el ocio.

- Medir la calidad ambiental.

- Conocer su satisfacción con la vida.

- Conocer el nivel educativo.

- Conocer el nivel de ingresos.

- Conocer la frecuencia de utilización de los servicios sociales y sanitarios, así como la satisfacción con dichos servicios.

- Valorar el deterioro cognitivo.

- Analizar, tanto en el grupo experimental como en el grupo control, la relación existente entre las siguientes variables: satisfacción con la vida, educación, ingresos, deterioro cognitivo, satisfacción con las relaciones, habilidades funcionales, frecuencia de las relaciones, salud objetiva, salud subjetiva y frecuencia de las actividades.

\section{- METODOLOGÍA}

La muestra seleccionada está formada por usuarios del centro social de personas mayores del ayuntamiento de Cée (A Coruña) y que acuden asiduamente a sus actividades. La evaluación se realizó desde el mes de septiembre al mes de junio por ser el período de tiempo en el cual se realizan las actividades en el centro, ya que durante la época estival no se llevan a cabo actividades. La primera evaluación se hizo en el mes de septiembre de 2011; la segunda, en febrero de 2012, y la tercera, en el mes de junio de 2012, habiendo transcurrido 5 meses entre cada una de ellas y 10 meses entre la primera y la última evaluación.

En cuanto a la muestra seleccionada para el grupo control, está formada por sujetos con características similares a las de los usuarios del centro social pero que no reciben ningún tipo de tratamiento gerontológico.

Tanto el grupo experimental como el control quedaron compuestos por 30 usuarios con un rango de edad comprendido entre los 60 y los 90 años.

Para confirmar que la calidad de vida de las personas mayores usuarias de un centro social es mejor que la de aquellos sujetos que no lo utilizan, tendríamos que considerar varios aspectos: por un lado, los subjetivos, y por otro los objetivos. Asimismo, debemos tener en cuenta además los estados social y emocional.

Por todo ello, hemos escogido para la evaluación de la calidad de vida el Cuestionario breve de la calidad de vida (CUBRECAVI) ${ }^{1}$, ya que ha demostrado ser una herramienta fiable y válida para la evaluación de la calidad de vida de forma multidimensional, además de ser un cuestionario de fácil aplicación, de duración breve (unos 20 minutos aproximadamente), y que mide las siguientes áreas: salud (salud subjetiva, objetiva y psíquica), integración social, habilidades funcionales, actividad y ocio, calidad ambiental, satisfacción con la vida, educación, ingresos, y servicios sociales y sanitarios.

Del mismo modo, nos hemos propuesto evaluar la existencia o no de deterioro cognitivo para ver si influye o no en la calidad de vida de los sujetos. Para ello hemos escogido el Cuestionario de estado mental portátil de Pfeiffer por ser un test fiable, de fácil aplicación y de corta duración (unos 4 minutos) y al que no le afecta el nivel cultural de los sujetos.

En un primer momento haremos un análisis descriptivo comparando los dos grupos que componen este estudio, hablando en puntuaciones medias y en algunos casos en porcentajes. Posteriormente, realizaremos un análisis de correlación de Pearson para analizar la relación existente entre las variables que más nos interesan utilizadas en este estudio, tanto en el grupo experimental como en el grupo control.

\section{- RESULTADOS}

En cuanto al nivel educativo predominante en los participantes de este estudio sobresale en ambos grupos "menos de estudios primarios pero sabían leer".

Si nos centramos en el nivel de ingresos mensuales, pudimos observar cómo en el grupo experimental destaca un 20\% que cobraba entre 301 y 450 euros, y un 30\% entre 451 y 600 euros. En el caso de los componentes del grupo control, destaca un 33,33\% que cobraba entre 301 y 450 euros, y un 26,67\% entre 451 y 600 euros.

En cuanto a las áreas de salud (subjetiva, objetiva y psíquica), integración social, habilidades funcionales, actividad y ocio, calidad ambiental, satisfacción con la vida, satisfacción y frecuencia de utilización de los servicios sociales y sanitarios, los usuarios del centro social han puntuado mejor que los componentes del grupo control, de modo que los usuarios del centro manifestaron: tener una mayor salud; estar más integrados socialmente; poder valerse mejor por sí mismos, así como tener menos dificultades para realizar las actividades básicas de la vida diaria; tener un mayor nivel de actividad; sentirse más satisfechos con la forma en la que ocupaban su tiempo; sentirse más satisfechos tanto con los diferentes aspectos objetivos de sus viviendas como con sus viviendas en general; sentirse más satisfechos con sus vidas, y utilizar con mayor frecuencia los servicios sociales y sanitarios así como sentirse más satisfechos con dichos servicios.

En cuanto al apartado del CUBRECAVI en el que se pretendía valorar la variable calidad de vida, observamos que las elecciones realizadas por los sujetos consultados, tanto de los usuarios del centro social como de los no usuarios, ponen de manifiesto un importante acuerdo en lo que se refiere a dos elementos cuya relevancia sobresale entre todos los demás, como son: "tener una buena salud" y "poder valerse por sí mismos". En concreto, en nuestro estudio aparece mayoritariamente, en ambos grupos, como primera opción la variable "tener una buena salud" y, como segunda opción, la variable "poder valerse por sí mismo”. Por otra parte, hemos comprobado cómo las variables "mantenerse activo", "tener buenos servicios sociales y sanitarios", "mantener buenas relaciones sociales y familiares", "sentirse satisfecho con la vida" y "tener una buena pensión o renta" son los ingredientes de la calidad de vida que aparecen seleccionados por los mayores en un segundo lugar.

En cuanto al estado cognitivo, en el grupo experimental ninguno de sus miembros tenía deterioro cognitivo; sin embargo, en el grupo control aparece en la primera evaluación un $10 \%$ de sus miembros con un deterioro moderado, para disminuir a un 3,33\% en las dos posteriores evaluaciones.

\section{ANÁLISIS DE CORRELACIÓN}

A continuación procederemos a analizar la relación existente entre las variables utilizadas en este estudio que más nos interesan, tanto en el grupo experimental como en el grupo control. Dichas variables son las siguientes: "satisfacción con la vida", "educación", "ingresos", "deterioro cognitivo", "apoyo social", "habilidades funcionales", "salud objetiva", "salud subjetiva" y "frecuencia de las actividades".

Procederemos a comentar las correlaciones más interesantes para nuestro estudio halladas entre las variables analizadas tanto en el grupo experimental como en el grupo control:

- En el caso de la variable "habilidades funcionales" apreciamos que mantiene una correlación positiva con la variable "salud objetiva" (tablas 1 y 2), con la variable "salud subjetiva" (tablas 1 y 2) y con la variable "frecuencia de las actividades" (tablas 1 y 2), es decir, los 
sujetos con un mayor nivel de habilidades funcionales tienen una mayor salud objetiva, una mayor salud subjetiva, y realizan actividades con mayor frecuencia.

- De igual modo, apreciamos una relación positiva entre las variables "apoyo social" y "salud subjetiva (tablas 2 y 3), de modo que existe una tendencia que nos indica que las personas con mayor salud subjetiva tienen un mayor apoyo social.

- Asimismo, apreciamos una tendencia positiva entre las variables "salud objetiva" y "salud subjetiva (tablas 1 y 2), es decir, los sujetos con mayor salud objetiva tienen una mayor salud subjetiva.

- En el caso de las variables "frecuencia de las actividades" y "salud subjetiva" apreciamos una relación positiva (tablas 1 y 2), de modo que podemos interpretar que las personas que realizan actividades con mayor frecuencia tienen una mayor salud subjetiva.

Además de las mencionadas correlaciones comunes a ambos grupos, debemos mencionar que en el caso del grupo de los sujetos que no asistían al centro social encontramos a mayores las siguientes correlaciones:

- Apreciamos que la variable "habilidades funcionales" mantiene una correlación positiva con la variable "educación" (tabla 3) y con la variable "apoyo social" (tabla 3), es decir, los sujetos con mayores habilidades funcionales tienen un mayor nivel educativo y un mayor apoyo social.

Tabla 1. Correlaciones en el grupo control

\begin{tabular}{|c|c|c|c|c|c|}
\hline & & $\begin{array}{l}\text { Habilidades } \\
\text { funcionales* }\end{array}$ & $\begin{array}{l}\text { Frecuencia de } \\
\text { las relaciones }\end{array}$ & Salud objetiva & Salud subjetiva \\
\hline & Correlación de Pearson & $-0,665$ & 0,186 & 0,558 & 0,444 \\
\hline $\begin{array}{l}\text { Frecuencia de las } \\
\text { actividades }\end{array}$ & Sig. (bilateral) & 0,000 & 0,326 & 0,001 & 0,014 \\
\hline & N & 30 & 30 & 30 & 30 \\
\hline & Correlación de Pearson & $-0,609$ & 0,135 & 1 & 0,572 \\
\hline Salud objetiva & Sig. (bilateral) & 0,000 & 0,479 & & 0,001 \\
\hline & N & 30 & 30 & 30 & 30 \\
\hline & Correlación de Pearson & $-0,459$ & $0,016^{c}$ & 0,572 & 1 \\
\hline Salud subjetiva & Sig. (bilateral) & 0,011 & 0,934 & 0,001 & \\
\hline & $\mathrm{N}$ & 30 & 30 & 30 & 30 \\
\hline
\end{tabular}

Las correlaciones significativas que analizaremos aparecen en negrita. Sig.: significación.

*La variable "habilidades funcionales" ha sido valorada en una escala con valor inverso al resto de las variables, de modo que las correlaciones de dicha variable deben de ser interpretadas de manera inversa al signo que tenga.

Tabla 2. Correlaciones en el grupo experimental

\begin{tabular}{|l|l|c|c|c|c|}
\hline & Correlación de Pearson & $\begin{array}{c}\text { Satisfacción con } \\
\text { las relaciones }\end{array}$ & $\begin{array}{c}\text { Habilidades } \\
\text { funcionales* }\end{array}$ & Apoyo social & Salud objetiva \\
\hline & Sig. (bilateral) & 0,150 & $-\mathbf{0 , 6 1 1}$ & $0,277^{*}$ & 1 \\
\hline & N & 0,428 & 0,000 & 0,139 & 30 \\
\hline & Correlación de Pearson & 30 & 30 & 30 & $\mathbf{0 , 5 1 9}$ \\
\hline & Sig. (bilateral) & 0,351 & $-\mathbf{0 , 6 1 5}$ & $\mathbf{0 , 3 8 3}$ & 0,003 \\
\hline & N & 0,057 & 0,000 & 0,037 & 30 \\
\hline
\end{tabular}

Las correlaciones significativas que analizaremos aparecen en negrita. Sig.: significación.

*La variable "habilidades funcionales" ha sido valorada en una escala con valor inverso al resto de las variables, de modo que las correlaciones de dicha variable deben de ser interpretadas de manera inversa al signo que tenga. 
Tabla 3. Correlaciones en el grupo control

\begin{tabular}{|l|l|c|c|c|}
\hline & $\begin{array}{l}\text { Correlación } \\
\text { de Pearson }\end{array}$ & $-\mathbf{0 , 4 3 9}$ & $-0,241$ & $\begin{array}{r}\text { Apoyo } \\
\text { social }\end{array}$ \\
\hline $\begin{array}{l}\text { Habilidades } \\
\text { funcionales* }\end{array}$ & Sig. (bilateral) & 0,015 & 0,217 & 0,038 \\
\hline & N & 30 & 28 & 30 \\
\hline & $\begin{array}{l}\text { Correlación } \\
\text { de Pearson }\end{array}$ & 0,406 & $-0,019$ & $\mathbf{0 , 4 9 1}$ \\
\hline \multirow{2}{*}{$\begin{array}{l}\text { Salud } \\
\text { subjetiva }\end{array}$} & Sig. (bilateral) & 0,026 & 0,925 & 0,006 \\
\hline & N & 30 & 28 & 30 \\
\hline
\end{tabular}

Las correlaciones significativas que analizaremos aparecen en negrita. Sig.: significación.

"La variable "habilidades funcionales" ha sido valorada en una escala con valor inverso al resto de las variables, de modo que las correlaciones de dicha variable deben de ser interpretadas de manera inversa al signo que tenga.

Tabla 4. Correlaciones en el grupo experimental

\begin{tabular}{|c|c|c|c|c|}
\hline & $\begin{array}{l}\text { Salud } \\
\text { subjetiva }\end{array}$ & $\begin{array}{l}\text { Frecuencia } \\
\text { de las } \\
\text { actividades }\end{array}$ & $\begin{array}{c}\text { Satisfacción } \\
\text { con la vida }\end{array}$ \\
\hline \multirow{3}{*}{$\begin{array}{l}\text { Salud } \\
\text { subjetiva }\end{array}$} & $\begin{array}{l}\text { Correlación } \\
\text { de Pearson }\end{array}$ & 1 & 0,415 & 0,156 \\
\hline & Sig. (bilateral) & & 0,023 & 0,411 \\
\hline & $\mathrm{N}$ & 30 & 30 & 30 \\
\hline
\end{tabular}

\section{- DISCUSIÓN}

De acuerdo con los resultados obtenidos, se determinó que al igual que propusimos en los objetivos, los usuarios del centro social manifestaron una mayor salud que los sujetos que no utilizaban los servicios del centro; de igual modo, mostraron estar más integrados socialmente, más activos, y funcionalmente más autónomos que los mayores que no acudían a dicho centro; esto viene a corroborar lo que defendía Villalobos ${ }^{2}$ de que los adultos mayores que acuden a dichos centros son más participativos y activos.
Asimismo, hemos comprobado que los usuarios del centro social apenas sufren deterioro cognitivo; sin embargo, los que no acuden a él tienen un deterioro superior.

Del mismo modo, los mayores del centro social manifestaron sentirse más satisfechos con sus vidas que los mayores que no acuden al centro: esto está en consonancia con lo que defendía Rojas ${ }^{3}$ en su investigación sobre la calidad de vida y autonomía, en la que expuso que en general los adultos perciben un sentimiento de bienestar fortalecido por ser personas independientes y funcionales.

Así, hemos comprobado que tanto en los usuarios del centro social como en los que no acuden a él, la valoración subjetiva de la salud estaba relacionada con las habilidades funcionales, con el apoyo social y con el nivel de actividad. En estas tres relaciones coincidimos con Azpiazu Garrido $^{4}$, quien también encontró asociaciones entre un mal estado de salud autopercibido y el nivel educativo y el nivel de ingresos.

Del mismo modo, hemos apreciado en ambos grupos una correlación entre las habilidades funcionales y la salud y la frecuencia de las actividades. Sin embargo, también observamos que en el caso de los mayores que no utilizaban los servicios del centro social, las habilidades funcionales también presentaban una correlación con otras variables, como el nivel educativo o el apoyo social. Este último punto, que no se ha podido verificar en el grupo experimental, ha sido resaltado por Céspedes ${ }^{5}$, quien defendía que cuanto más autónomos sean los gerontes mayor soporte social presentan.

De igual modo, no hemos encontrado relación entre "deterioro cognitivo" y "habilidades funcionales"; esto puede ser debido a que los participantes de nuestro estudio apenas presentaban deterioro cognitivo. En concreto, los usuarios del centro social no presentaban deterioro, y en el caso de los no usuarios sólo unos pocos presentaron deterioro. Sin embargo, podemos mencionar que en el estudio de Mora, Villalobos, Araya y $\mathrm{Ozols}^{6}$ sí se encontró relación entre las habilidades funcionales y el deterioro cognitivo.

Asimismo, hemos hallado en ambos grupos una relación significativa entre la salud subjetiva y la salud objetiva. Esta relación puede ser debida, como dijo Fernández-Ballesteros ${ }^{7}$, a que una mala valoración de la propia salud puede surgir como respuesta ante la toma de conciencia de que se sufre una enfermedad, o bien como reacción al dolor o impacto de la enfermedad sobre la vida diaria.

Del mismo modo, cabe resaltar que no hemos encontrado en nuestro estudio relación entre la satisfacción con la vida y el nivel de ingresos del mayor; sin embargo, en el estudio de Mendoza Sierra ${ }^{8}$ sí se aprecia un aumento de la satisfacción con la vida conforme los ingresos económicos son mayores

\section{- BIBLIOGRAFÍA}

1. Fernández-Ballesteros R, Zamarrón MD. Cubrecavi. Cuestionario breve de la calidad de vida. Madrid: TEA Ediciones; 2007.

2. Villalobos D. Problemas de salud que enfrenta la población de la tercera edad en la ciudad de Heredia. Tesis para optar al título de Magíster en Gerontología. Facultad de Medicina, Universidad de Costa Rica, San José, Costa Rica; 1989.

3. Rojas L. Calidad de vida y autonomía en personas mayores. Tesis para la obtención del título de Magíster en Gerontología. Facultad de Medicina, Universidad de Costa Rica, San José, Costa Rica; 1999.
4. Azpiazu Garrido M, Cruz Jentoft A, Villagrasa Ferrer R, Abanades Herranz JC, García Marín N, Alvear Valero de Bernabé F. Factores asociados a mal estado de salud percibido o mala calidad de vida en personas mayores de 65 años. Revista Española de Salud Pública 2002; 6: 683-700.

5. Céspedes A. Influencia de los factores socioeconómicos en la pérdida de autonomía de los adultos mayores costarricenses entre los 65 y 80 años. Programa de investigación sobre el envejecimiento. San José, Costa Rica: Universidad de Costa Rica; 1987
6. Mora M, Villalobos D, Araya G, Ozols A. Perspectiva subjetiva de la calidad de vida del adulto mayor, diferencias ligadas al género y a la práctica de la actividad físico recreativa. Revista MHSalud 2004;1:1.

7. Fernández-Ballesteros R, Izal M. Introducción a la evaluación psicológica. Madrid: Pirámide; 1992.

8. Mendoza Sierra MI, Revilla Delgado C, Feria Muñoz A, Bernal Vilán JM, Barbero Prado M, Revilla Parody T. La calidad de vida de las personas mayores en una zona de salud de Huelva. Portularia 2004; 4: 199-208. 